\title{
Correspondence
}

\section{Malignant hyperthermia: choice of anaesthesia for muscle biopsy}

To the Editor:

With reference to the review on malignant hyperthermia by Britt, ${ }^{1}$ we were curious why muscle biopsies would be done using general anaesthesia. At the Ottawa Civic Hospital, muscle biopsies are taken from the vastus lateralis muscle during spinal anaesthesia with tetracaine, or with the use of femoral and lateral femoral cutaneous nerve blocks with 2-chloroprocaine.

Over 30 muscle biopsies have been done to date in our hospital, with no adverse reactions. Recently a protocol has been proposed for performing muscle biopsies under femoral nerve block in conjunction with lateral femoral cutaneous nerve block, using amide anaesthetics. ${ }^{2}$ The advantages of regional anaesthesia in these high-risk patients are simplicity, safety, patient acceptance and surgical conditions which do not interfere with the diagnostic tests.

Geraint W. Lewis MB, BCH, FRCPC

Charles Cattran MD, FRCPC

Department of Anaesthesia

Ottawa Civic Hospital

Ottawa, Ontario

\section{REFERENCES}

1 Britt BA. Malignant hyperthermia. Can Anaesth Soc J 1985; 32: 666-77.

2 Berkowitz A, Rosenberg $H$. Femoral block with mepivacaine for muscle biopsy in malignant hyperthermia in patients. Anesthesiology 1985; 62: 651-2.

\section{REPLY}

I am grateful for the opportunity to respond to the letter of Doctors Lewis and Cattran.

In Toronto, diagnostic tests performed in the Malignant Hyperthermia Investigation Unit at the University of Toronto are from muscle excised in both the Toronto
General Hospital and the Mount Sinai Hospital. For these biopsies, general anaesthesia is used in the former institution, and femoral nerve plus lateral femoral cutaneous nerve blocks in the latter hospital. Patients presenting in the Toronto General Hospital who do not wish to have a general anaesthetic are offered the option of transferring to the Mount Sinai Hospital. Not many, however, desire to do so. The majority not only prefer a pain-free biopsy, but also a stress-free biopsy.

Onty the caffeine-halothane contracture test and routine microscopy are performed on muscle obtained from patients undergoing biopsy in the Mount Sinai Hospital and also in the Ottawa Civic Hospital. On the other hand, muscle removed from Toronto General Hospital patients is utilized not only for the caffeine-halothane contracture test and routine microscopy but also for a number of other diagnostic screening and research tests. These include the skinned fibre test, ATP depletion, calcium uptake into thin muscle sections, calcium-induced calcium release from single skinned fibres and influence of phospholipase, phospholipase inhibitors, and other drugs on caffeine andior halothane contractures. Obviously, much more muscle must be excised in order to do all these extra experiments - experiments that significantly improve accuracy of diagnosis. Few patients would care to part with such an amount of muscle while awake.

We have also noted that centres performing muscle biopsy for diagnosis of $M H$ with the aid of regional anaesthesia often use considerable amounts of supplementing sedation, including diazepam, fentanyl and nitrous oxide. The amounts used are sometimes large enough to depress respiration sufficiently to induce a rise in the concentration of carbon dioxide and a fall in the concentration of oxygen in the tissues, changes that certainly must have an adverse effect on the muscle. Furthermore, the stress which is certainly experienced by most patients undergoing regional anaesthesia induces release of catecholamines, which also may interfere with other test results.

Although Lewis and Cattran cite 30 consecutive uncomplicated biopsies as a reason to prefer regional to general anaesthesia, I feel that they are being rather too sanguine. We have performed (as of February 1986), over 1100 biopsies and have observed several sequences of over 100 consecutive biopsies without complication, only to observe a problem such as mild fever in the recovery room on a subsequent anaesthetic. When we first began doing these biopsies, they were performed eirher with epidural, local or regional anaesthesia. Several of these patients also experienced fever following their surgery. Additionally, many complained that the procedure had been extremely unpleasant and painful. It was at least in part because of these complaints that we decided to change to general anaesthesia. 
Finally, I would like to bring to the attention of Journal readers a report' to be presented at the 1986 CAS Annual Meeting in Montréal. This paper summarizes our experience with 956 patients having muscle biopsies between 1968 and 1984 and demonstrates that only 0.6 per cent of the patients developed $M H$ reactions, which were invariably mild and quite amenable to dantrolene therapy.

Beverley A. Britt MD, FRCPC

Department of Anaesthesia

University of Toronto

Toronto, Ontario

\section{REFERENCE}

1 Cunliffe $M$, Lerman $J$, Britt BA. Is prophylactic dantrolene indicated for MHS patients undergoing elective surgery? Can Anaesth Soc J 1986; 33: $\$ 113-S 114$

\section{Magne ic resonance imaging}

To the Editor:

I was interested to read the CME section on Imaging for Anaesthestists by Weston et al. ${ }^{1}$ There are several points which I feel are worthy of mention with regard to magnetic resonance imagers (MRI).

First, as the imaging coil is typically two metres in length, with an intemal diameter of 0.6 metres, the patient is not only remote from the anaesthetist, but also inaccesible, poorly illuminated and often completely hidden from view. Further, enhanced resolution for cranial imaging may require placement of the patient's head inside a supplementary coil. The additional space limitations imposed under these circumstances requires the use of preformed plastic tubes (e.g. Rae), if endotracheal anaesthesia is to be administered. These problems are not unique, particularly in the radiology environment, but imply we must depend heavily on monitoring systems, which may themselves be subject to magnetic or radiofrequency interference. The enclosed environment leads to claustrophobia in three to four per cent of awake patients, ${ }^{2}$ whilst the drumming noise emitted by MRI devices during scanning may be uncomfortable for some patients. Sedation may not have the desired effect in these patients.

Second, superconducting toroidal magnets as used in some MRI devices are unstable. They are kept at an operating temperature of $-270 \mathrm{~K}$ by liquid nitrogen and helium, and cannot be switched off. The static magnetic field effects are therefore ever present and precautions relating to ferrous containing devices apply at all times, even in an emergency. MRI devices using resistive magnets may be switched off in an emergency, but may take several hours to stabilise when restarted.

Third, the current generation of MRI devices operate at a magnetic field strength of $0.15-0.5$ Tesla, but the new devices are capable of developing field strengths up to 2 Tesla. Shielding is required to control the extent of the magnetic field outside the imaging room with these high field strengths and the anaesthetist must be familiar with his local imager before embarking on anaesthesia. As in many other areas, often "just a whiff of gas" is not quite as straightforward as it appears to our colleagues from other specialities.

\section{Nixon MB, CH B, FFARCS}

Department of Anaesthesia

University College Hospital

London, England

\section{REFERENCES}

1 Weston $G$, Strunin L, Amundson GM. Imaging for Anaesthetists: a review of the methods and anaesthetic implications of diagnostic imaging techniques. Can Anaesth Soc J 1985; 32: 552-61.

2 Bydder $G M$. Clinical nuclear magnetic resonance imaging. Br J Hosp Med 1983; 29: 348-56. 